\title{
ANÁLISIS ENERGÉTICO DE CUATRO SECUENCIAS DE CULTIVO EN LA PROVINCIA DE BUENOS AIRES, ARGENTINA
}

\section{ENERGETIC ANALYSIS OF FOUR CROP SEQUENCES IN BUENOS AIRES PROVINCE, ARGENTINA}

\author{
Adriana M. Chamorroo ${ }^{1 *}$, Silvina I. Golik ${ }^{1}$, Rodolfo Bezus ${ }^{1}$, Andrea E. Pellegrini ${ }^{1}$ \\ ${ }^{1}$ Facultad de Ciencias Agrarias y Forestales, Universidad Nacional de La Plata, Calle 60 y 119, C.C.31. \\ C.P. 1900, La Plata. Pcia. de Buenos Aires, Argentina. \\ * Autor para correspondencia E-mail: chamorro@agro.unlp.edu.ar
}

\section{RESUMEN}

El uso de la energía fósil en la agricultura es importante porque es un recurso escaso, no renovable, que debe ser conservado para las generaciones futuras, y su consumo se ha relacionado con el calentamiento global del planeta. El objetivo de este trabajo fue analizar los flujos de energía y evaluar el balance de energía (BE) y la eficiencia energética (EE) de cuatro secuencias (rotaciones) de cultivo: avena/soja (Avena sativa L./Glycine max (L.) Merr.), cebada/soja (Hordeum distichun L.)/Glycine max (L.) Merr.), colza/soja (Brassica napus L.)/Glycine max (L.) Merr.) y trigo/soja (Triticum aestivum L.)/ Glycine max (L.) Merr.), producidas bajo dos manejos tecnológicos en Buenos Aires, Argentina. La energía ingresada a las secuencias fue similar en las dos campañas evaluadas (13,8 GJ ha-1) pero el BE y la EE fueron mayores en la primera por las mejores condiciones meteorológicas. Los resultados mostraron diferentes flujos energéticos, BE y EE en las secuencias evaluadas. La EE fue elevada (9,4$17,2)$ decreciendo en el orden cebada/soja-trigo/soja-avena/soja-colza/soja. La adaptación agroecológica diferencial de los cultivos y el "efecto antecesor" sobre la soja explicarían las diferencias. El manejo tecnológico tuvo menor influencia sobre las variables energéticas que los cultivos que componen la secuencia y las condiciones de crecimiento. El mejor comportamiento lo registró la secuencia cebada/ soja, que logra alta EE y BE con gastos energéticos poco más altos que las otras secuencias. Se considera importante evaluar integralmente estas opciones productivas incorporando otros indicadores de sustentabilidad ecológica así como aspectos económicos y sociales de la sustentabilidad.

Palabras clave: avena, soja, cebada, colza, trigo, rotación de cultivos, eficiencia energética.

\section{ABSTRACT}

The use of fossil energy in agriculture is an important issue. In fact, fossil energy is a scarce and non-renewable resource, which needs to be preserved for future generations but whose consumption has been linked to global warming. The aim of this work was to analyze the energy flows and evaluate the energy balance (BE) and energy efficiency (EE) of four crop sequences (rotations): oat (Avena sativa L.)/soybean (Glycine max (L.) Merr.), barley (Hordeum distichun L.)/soybean, rapeseed (Brassica napus L.)/soybean, wheat (Triticum aestivum L.)/soybean, produced under two different levels of technological management in Buenos Aires (Argentina). Incoming energy to crop sequences was similar in the two seasons evaluated $\left(13.8 \mathrm{GJ} \mathrm{ha}^{-1}\right)$ but $\mathrm{BE}$ and EE were greater in the first season due to better meteorological conditions. Results showed different energy flows, BE and EE in the evaluated sequences. Energy efficiency was high (9.4-17.2) and values decreased gradually for barley/ 
soybean-wheat/soybean-oat/soybean-rapeseed/soybean. The use of a different agro-ecological crop adaptation strategy and the 'predecessor effect' on soybean production would count for these differences. Technological management had a lower influence on energy variables compared to crops and growth conditions. Barley/soybean showed the best performance due to its high EE and BE, presenting energy costs that were only a little higher than the other sequences. It is important to fully evaluate these productive options by incorporating other indicators of ecological sustainability, as well as economic and social aspects of sustainability.

Key words: oat, soybean, barley, rapeseed, wheat, energy efficiency.

\section{INTRODUCCIÓN}

La agricultura moderna ha experimentado importantes cambios con el objeto de incrementar la producción y cubrir una demanda de alimentos cada vez mayor, resultante del crecimiento poblacional y de cambios en los estilos de vida de las personas (FAO, 2011). Entre ellos, se ha producido una intensificación del aporte de insumos, los que pueden ser expresados en términos de energía, ya que la requieren para su producción. El aporte creciente de energía fósil a la producción agrícola ha cobrado renovada atención no sólo porque se trata de un recurso limitado que debe ser conservado para las futuras generaciones, sino también porque el mayor consumo de combustible en la agricultura, y el consecuente incremento en la emisión de dióxido de carbono a la atmósfera, ha sido relacionado con el calentamiento global del planeta (Tzilivakis et al., 2005). Por lo tanto, un uso eficiente de la energía es una importante condición que hace a la sustentabilidad de los sistemas agrícolas (Altieri y Nicholls, 2000; Tzilivakis et al., 2005; Gliessman et al., 2007; Deike et al., 2008, Zentner et al., 2011).

El uso de la energía en los agroecosistemas se puede evaluar a través del análisis de sus flujos de energía (entradas y salidas), y de la relación entre la energía contenida en los productos cosechados y la energía necesaria para su producción, lo que se conoce como eficiencia energética. En un escenario de escasez energética es necesario el análisis de la energía requerida para la producción de los distintos bienes (en este caso, productos agrícolas), ya que no sólo es importante que la producción sea energéticamente eficiente, sino que necesite montos de energía (principalmente no renovable) relativamente bajos (Altieri y Nicholls, 2000). Igualmente y dado que, por definición, el objetivo de los agroecosistemas es producir un flujo constante de bienes y servicios, es necesario que sean suficientemente productivos, aspecto que suele ser evaluado a través del balance de energía, esto es, la diferencia entre la energía que sale del agroecosistema como productos de cosecha y la que ingresa a través de los insumos (Moreno et al., 2011).
El sector agropecuario argentino ha experimentado significativos cambios en las últimas décadas, relacionados con la fuerte expansión del cultivo de la soja y su paquete tecnológico, que han dado en llamarse "sojización" y "pampeanización", y han conducido a un creciente deterioro ambiental, concentración económica, exclusión social y pérdida de soberanía alimentaria (Pengue, 2004). El partido de Magdalena, en el área de influencia de la Facultad de Ciencias Agrarias y Forestales de la Universidad Nacional de La Plata, se caracteriza por ser de producción mixta (agrícola-ganadera), por lo cual, la soja se ha difundido más lentamente, pero no queda exenta a esta problemática. Con el fin de evitar o minimizar en esta zona los problemas ambientales y sociales asociados al monocultivo sojero se considera necesario generar información local que permita incluir a este cultivo de manera más racional en los esquemas productivos zonales. Una primera aproximación implica evaluar distintas secuencias (rotaciones) que la incluyan, y a la vez incrementen la diversidad de cultivos intervinientes en las mismas.

Una forma ampliamente difundida de sembrar la soja es bajo la modalidad de soja de segunda, lo que significa sembrarla inmediatamente después de la cosecha de un cultivo de invierno, sin mediar un período de barbecho, pero usualmente un poco retrasada de su fecha óptima de cosecha. Esto, aunque penaliza los rendimientos de la soja, permite obtener dos cosechas en un año. El cultivo invernal tradicionalmente usado en este planteo de doble cultivo es el trigo (Triticum aestivum $\mathrm{L}$.), pero otras especies pueden cumplir esa función. Una de ellas es la cebada (Hordeum distichun L.), cuyos granos se destinan a la producción de malta para la industria cervecera. Su área cultivada ha crecido notablemente en la Argentina en los últimos 20 años, acompañando el crecimiento de la producción de cerveza y favorecida por la posibilidad de adelantar la siembra de la soja de segunda en aproximadamente 15 días, ya que el ciclo de la cebada es más corto comparado con el del trigo, determinando la obtención de mejores rendimientos (Tomaso, 2011).

Otra opción es la avena (Avena sativa L.), muy 
importante en el país, principalmente como cultivo forrajero o de doble propósito, se pastorea durante el invierno y luego se deja semillar y se cosecha el grano. Sin embargo, recientemente se ha revalorizado su uso en la alimentación humana habiendo crecido la demanda de sus granos para la elaboración de cereales para el desayuno y galletas (Cortese, 2009).

La colza (Brassica napus L.) es otro antecesor posible para la soja de segunda. Es una oleaginosa perteneciente a la familia de las Crucíferas, lo que le otorga la posibilidad de facilitar el manejo de plagas, enfermedades y malezas propias de las gramíneas de invierno en sistemas productivos basados en estos cereales (Iriarte, 2009). Al igual que la cebada, normalmente se cosecha 10 a 15 días antes que el trigo, permitiendo la anticipación de la siembra de la soja de segunda. Su superficie en el país ha crecido alentada por la Ley Nacional 26093 de Biocombustibles sancionada en el año 2006 (Iriarte, 2009)

Debido a que la sustentabilidad en los sistemas productivos se plantea desde distintas dimensiones (ecológica, económica, social, cultural) (Sarandón et al., 2006), es necesario evaluar las distintas opciones de producción de una manera integral, no sólo desde una visión técnico-productiva. Las distintas opciones de doble cultivo planteadas han sido evaluadas en algunos aspectos de su producción (Chamorro et al., 2014a), sanidad (Golik et al., 2014a), balances de nutrientes (Golik et al., 2014b), eficiencia de uso del agua (Chamorro et al., 2014b), efectos sobre la bioquímica del suelo (Pellegrini et al., 2014). En este trabajo se plantea la evaluación de su comportamiento energético. Además, el resultado de los cultivos así como sus efectos sobre los agroecosistemas, no sólo dependen del ambiente sino también de cómo se realicen, es decir, de la tecnología aplicada, entendiendo como tal no sólo a los insumos utilizados sino también a los procesos por lo que es necesario analizar también distintas maneras posibles de producirlos.

Por lo anterior, el objetivo de este trabajo fue analizar los flujos de energía y evaluar comparativamente el balance de energía y la eficiencia energética de las secuencias avena/soja, cebada/ soja, colza/soja y trigo/soja, producidas bajo dos modelos tecnológicos en la provincia de Buenos Aires, Argentina.

\section{MATERIALES Y MÉTODOS}

Se condujo un experimento a campo en la Estación Experimental Julio Hirschhorn de la Facultad de Ciencias Agrarias y Forestales, de la Universidad Nacional de La Plata $\left(34^{\circ} 5^{\prime}\right.$ S, 57 $57^{\circ}$ O) en las campañas 2011-12 y 2012-13.
Los tratamientos fueron la combinación de cuatro secuencias de cultivo (avena/soja, cebada/ soja, colza/soja y trigo/soja) manejadas bajo dos modelos de producción: un nivel tecnológico medio (NTM), correspondiente al utilizado por la mayor parte de los productores de la zona, y un manejo identificado como nivel tecnológico alto (NTA), que es el implementado por aquellos productores que, consistentemente, a través de los años, obtienen mayores rendimientos en sus cosechas. Ambos manejos fueron conocidos a partir de entrevistas con productores y técnicos que se desempeñan en la zona. Estos tratamientos, en el ensayo, se plantearon en términos de materiales genéticos utilizados, dosis y tipo de fertilizantes aplicados, y utilización de fungicidas (para enfermedades foliares en los cereales y para Sclerotinia sclerotiorum en colza) ya que el (reducido) tamaño de las parcelas no permitió implementar diferencias respecto del manejo de insectos y malezas. El diseño experimental fue en bloques al azar con cuatro repeticiones y parcela dividida, correspondiendo la parcela mayor a la secuencia de cultivos y la menor $\left(22 \mathrm{~m}^{2}\right)$ al manejo tecnológico.

Para el análisis del flujo de la energía se siguió el modelo propuesto por Hülsbergen et al. (2001), por lo que sólo se contabilizó el ingreso de energía (IE) no renovable al sistema. En este modelo se contempla por un lado el ingreso de "energía directa", que es la contenida en el combustible utilizado en la producción de los cultivos, y por otro, el ingreso de "energía indirecta", que es aquella que ingresa al sistema a través de la fabricación de las maquinarias y los insumos. Para los cálculos, todas las entradas y salidas fueron convertidas en unidades equivalentes (GigaJoules, GJ) a partir de distintas fuentes bibliográficas. En la Tabla 1 se presentan los datos utilizados en los cálculos.

El IE se calculó en función de la cantidad de labores e insumos utilizados para realizar cada cultivo. El consumo de combustible para las labores se calculó utilizando el programa Costo Maq - Versión 1.1, Software para la gestión integral de la maquinaria agrícola (Donato et al., 2006). El consumo de combustible para la cosecha se calculó según Nagy (1999). Se adicionó también el costo energético de los lubricantes necesarios como 0,0036 GJ por cada litro de combustible usado (Dalgaard et al., 2001). La energía asociada a la construcción de la maquinaria se consideró linealmente relacionada con el consumo de combustible en cada labor, calculándose como 0,012 GJ por cada litro de combustible utilizado (Dalgaard et al., 2001). La energía indirecta ingresada a través de los insumos se relaciona en forma lineal con la dosis aplicada. En el caso de los fertilizantes y plaguicidas, la energía se calculó toman- 
Tabla 1. Costo energético de las labores y energía asociada a los insumos utilizados en este trabajo según distintos autores.

Table 1. Energetic cost of field operations and energy associated with inputs used in this study according to different authors.

\begin{tabular}{|c|c|c|c|c|c|}
\hline Concepto & $\begin{array}{r}\text { Coeficiente } \\
\text { energético }\end{array}$ & Autor ${ }^{\mathrm{a}}$ & Concepto & $\begin{array}{l}\text { Coeficiente } \\
\text { energético }\end{array}$ & Autor ${ }^{a}$ \\
\hline \multicolumn{3}{|l|}{ Labores (combustible) } & \multicolumn{3}{|l|}{ Insumos } \\
\hline Rastra de discos & 0,235 GJ ha-1 $^{-1}$ & 1 & Trifluralina & $0,167 \mathrm{GJ} \mathrm{L}^{-1}$ & 5 \\
\hline Rastra de dientes & 0,078 GJ ha-1 & 1 & Glifosato & $0,511 \mathrm{GJ} \mathrm{L}^{-1}$ & 2 \\
\hline \multirow{3}{*}{$\begin{array}{l}\text { Siembra grano fino } \\
\text { (con labranza) con } \\
\text { fertilización }\end{array}$} & \multirow[t]{3}{*}{$0,271 \mathrm{GJ} \mathrm{ha}^{-1}$} & \multirow[t]{3}{*}{1} & Cipermetrina & $0,580 \mathrm{GJ} \mathrm{L}^{-1}$ & 6 \\
\hline & & & D metoato & $0,229 \mathrm{GJ} \mathrm{L}^{-1}$ & 6 \\
\hline & & & Endosulfán & $0,355 \mathrm{GJ} \mathrm{L}^{-1}$ & 7 \\
\hline Siembra directa soja & 0,373 GJ ha-1 & 1 & Carbendazim & $0,397 \mathrm{GJ} \mathrm{L}^{-1}$ & 8 \\
\hline \multirow{2}{*}{$\begin{array}{l}\text { Aplicación de } \\
\text { plaguicidas }\end{array}$} & \multirow[t]{2}{*}{ 0,050 GJ ha-1 } & \multirow[t]{2}{*}{1} & Trifloxistrobin & $0,268 \mathrm{GJ} \mathrm{L}^{-1}$ & 9 \\
\hline & & & Cyproconazole & $0,268 \mathrm{GJ} \mathrm{L}^{-1}$ & 9 \\
\hline \multirow{2}{*}{$\begin{array}{l}\text { Aplicación de } \\
\text { fertilizante }\end{array}$} & \multirow[t]{2}{*}{ 0,136 GJ ha-1 } & \multirow[t]{2}{*}{1} & Nitrógeno (N) & $0,0756 \mathrm{GJ} \mathrm{kg}^{-1}$ & 5 \\
\hline & & & Fósforo (P2O5) & 0,0095 GJ kg-1 & 5 \\
\hline \multirow{2}{*}{$\begin{array}{c}\text { Cosecha cultivos } \\
\text { de invierno }\end{array}$} & \multirow[t]{2}{*}{$0,119 \mathrm{GJ} \mathrm{tn} \mathrm{tn}^{-1}$} & \multirow[t]{2}{*}{2} & Azufre (S) & $0,0011 \mathrm{GJ} \mathrm{kg}^{-1}$ & 5 \\
\hline & & & Grano de avena & $0,0194 \mathrm{GJ} \mathrm{kg}^{-1}$ & 2 \\
\hline Cosecha soja & 0,083 GJ tn-1 & 3 & Grano de cebada & $0,0185 \mathrm{GJ} \mathrm{kg}^{-1}$ & 2 \\
\hline \multicolumn{3}{|l|}{ Insumos } & Grano de colza & $0,0294 \mathrm{GJ} \mathrm{kg}^{-1}$ & 2 \\
\hline Semilla de avena & $0,0072 \mathrm{GJ} \mathrm{kg}^{-1}$ & 2 & Grano de trigo & $0,0187 \mathrm{GJ} \mathrm{kg}^{-1}$ & 2 \\
\hline Semilla de cebada & $0,0072 \mathrm{GJ} \mathrm{kg}^{-1}$ & 2 & Grano de soja & $0,0169 \mathrm{GJ} \mathrm{kg}^{-1}$ & 4 \\
\hline Semilla de colza & $0,0138 \mathrm{GJ} \mathrm{kg}^{-1}$ & 2 & & & \\
\hline Semilla de trigo & $0,0072 \mathrm{GJ} \mathrm{kg}^{-1}$ & 2 & & & \\
\hline Semilla de soja & $0,0169 \mathrm{GJ} \mathrm{kg}^{-1}$ & 4 & & & \\
\hline Dicamba & $0,315 \mathrm{GJ} \mathrm{L}^{-1}$ & 5 & & & \\
\hline Metsulfurón & $0,351 \mathrm{GJ} \mathrm{L}^{-1}$ & 5 & & & \\
\hline
\end{tabular}

a 1: Donato et al., 2006; 2: Nagy, 1999; 3: Donato et al., 2008; 4: Borin et al., 1997; 5: Zentner et al., 2004; 6: Guzmán y Alonso, 2008; 7: Iermanó y Sarandón, 2009; 8: Tzilivakis et al., 2005; 9: Khaledian et al., 2010.

do en cuenta la concentración del nutriente o el principio activo en el producto comercial, la dosis aplicada y el contenido energético del principio activo o el nutriente de acuerdo a la bibliografía consultada. La salida de energía para cada producto se calculó relacionando el rendimiento del mismo y su contenido energético.

Se calculó la eficiencia energética (EE) como la relación entre la energía de origen no renovable ingresada al sistema y las salidas de energía para cada producto, y el balance de energía (BE) como la diferencia entre las salidas y las entradas de energía para cada situación.

Los datos se procesaron por el análisis de la varianza (ANOVA) usando la prueba de Tukey ( $\mathrm{P}$ $<0,05$ ) para la separación de medias.

\section{RESULTADOS}

Desarrollo de los ensayos y producción de los cultivos

Los ensayos se desarrollaron bajo diferentes condiciones meteorológicas que determinaron diferentes niveles de producción de los cultivos. En la campaña 2011-12, aunque los cultivos de invierno recibieron pocas precipitaciones durante su floración o antesis, contaron con agua acumulada en el perfil del suelo que les permitió alcanzar rendimientos elevados: $6560 \mathrm{~kg} \mathrm{ha}^{-1}$ para avena, $9030 \mathrm{~kg} \mathrm{ha}^{-1}$ para cebada, $3250 \mathrm{~kg} \mathrm{ha}^{-1}$ para colza y $8460 \mathrm{~kg} \mathrm{ha}^{-1}$ para trigo, y diferenciarse según la tecnología aplicada: $7262 \mathrm{~kg} \mathrm{ha}^{-1}$ y $6386 \mathrm{~kg}$ ha $^{-1}$ para el NTA y NTM, respectivamente. También la soja de segunda alcanzó altos rendimientos y se diferenció según el antecesor, rindió 4350 $\mathrm{kg} \mathrm{ha}{ }^{-1}$ sobre cebada y $4718 \mathrm{~kg} \mathrm{ha}^{-1}$ sobre colza, que liberaron antes el terreno, pero $3643 \mathrm{~kg} \mathrm{ha}^{-1}$ sobre avena y $3266 \mathrm{~kg} \mathrm{ha}^{-1}$ sobre trigo que fueron más tardíos, pero no se diferenció según el NT.

En la campaña 2012-13, durante la primavera se registraron precipitaciones excesivas que favorecieron el desarrollo de enfermedades, determinando menores rendimientos para los cultivos de invierno que, además, no se diferenciaron según el manejo tecnológico: $3154 \mathrm{~kg} \mathrm{ha}^{-1}$ para avena, $4746 \mathrm{~kg} \mathrm{ha}^{-1}$ para cebada, $2066 \mathrm{~kg} \mathrm{ha}^{-1}$ para colza y 
$6077 \mathrm{~kg} \mathrm{ha}^{-1}$ para trigo. La soja tuvo una producción algo mejor que en la campaña anterior y los rendimientos también se diferenciaron según el antecesor, pero de manera diferente a la campaña 2011-12: $4530 \mathrm{~kg} \mathrm{ha}^{-1}$ cuando se sembró sobre avena, $4848 \mathrm{~kg} \mathrm{ha}^{-1}$ sobre cebada, $43804 \mathrm{~kg} \mathrm{ha}^{-1}$ sobre colza y $3424 \mathrm{~kg} \mathrm{ha}^{-1}$ sobre trigo. Nuevamente, la producción de la soja no fue afectada por el NT.

\section{Los flujos de energía en las secuencias de cultivos}

Los resultados mostraron diferencias en los flujos energéticos de las secuencias de doble cultivo avena/soja, cebada/soja, colza/soja y trigo/soja, y también en el balance de energía y la eficiencia con que ésta fue usada.

También se verificó la influencia del ambiente a través de las condiciones meteorológicas del año sobre el comportamiento energético de los cultivos y las secuencias que componen.

Las tres variables más importantes desde el punto de vista de la sustentabilidad ecológica, el IE, el BE y la EE, se diferenciaron entre secuencias en las dos campañas (Tabla 2). En 2011-12, el IE registró interacción Secuencia x NT significativa pero en los dos NT el mayor IE correspondió a las secuencias trigo/soja y cebada/soja, y el menor a colza/soja.

En ambas campañas, la energía total ingresada a las distintas secuencias estuvo compuesta, en promedio, por $23,4 \%$ de energía directa (combustible), que fue significativamente mayor en cebada/soja y menor en colza/soja, debido a los rendimientos obtenidos en cada secuencia.

Respecto de la energía indirecta (asociada a los insumos y maquinarias), fue mayor en la secuencia trigo/soja y menor en colza/soja (Fig. 1). En ambos años y en todas las secuencias, los mayores gastos de energía indirecta los representaron los fertilizantes (43\%) y las semillas (33\%). Sin embargo, se observó que en la secuencia colza/ soja los aportes energéticos asociados a las semillas y a las maquinarias fueron más bajos que en las secuencias que incluyeron cereales (Fig. 1).

El BE fue mayor en la campaña 2011-12 que en 2012-13. En ambos casos, el mejor comportamiento lo mostraron las secuencias que incluyeron cebada y trigo, y la secuencia de menor producción fue colza/soja (Tabla 2). Como resultado, la secuencia cebada/soja fue la más eficiente y colza/ soja la de menor EE (Tabla 2).

El IE a los cultivos de invierno promedió 8,2 GJ ha ${ }^{-1}$ representando alrededor del $60 \%$ del total de energía ingresada a las secuencias. Además, registró interacción significativa Cultivo x NT en ambas campañas (Tabla 3) que, aunque careció de sentido biológico, se relacionó con diferencias en el ingreso de energía indirecta (Tabla 4).

La energía directa siguió la tendencia de los rendimientos, ya que gran parte del combustible se usó para la cosecha (Tabla 4). La energía indirecta en ambas campañas fue mayor en el NTA debido al alto costo energético de los fertilizantes. Las diferencias entre cultivos y entre años en el ingreso de energía indirecta se debieron a las distintas densidades de siembra y contenidos ener-

Tabla 2. Ingreso de energía (IE), balance de energía (BE) y eficiencia energética (EE) para cuatro secuencias de doble cultivo, promedio de dos manejos tecnológicos (NT), en dos temporadas, provincia de Buenos Aires, Argentina.

Table 2. Energy input (IE), energy balance (BE) and energetic efficiency (EE) for four crop sequences and average value for two levels of technological management (NT) in two seasons, Buenos Aires province, Argentina.

\begin{tabular}{|c|c|c|c|c|c|c|}
\hline \multirow{2}{*}{$\begin{array}{l}\text { Secuencia de } \\
\text { cultivos }\end{array}$} & \multicolumn{3}{|c|}{ 2011-12 } & \multicolumn{3}{|c|}{ 2012-13 } \\
\hline & IE & BE & EE & IE & BE & $\mathrm{EE}$ \\
\hline & \multicolumn{3}{|c|}{------ GJ ha-1 ------ } & \multicolumn{3}{|c|}{------ GJ ha-1 ------ } \\
\hline $\mathrm{Av} / \mathrm{S}$ & 14,2 & $189,4 \mathrm{~b}$ & $14,29 \mathrm{bc}$ & $13,9 \mathrm{~b}$ & $123,7 \mathrm{~b}$ & $9,96 \mathrm{~b}$ \\
\hline $\mathrm{Ce} / \mathrm{S}$ & 14,5 & 234,3 a & $17,19 \mathrm{a}$ & $13,8 \mathrm{~b}$ & $156,2 \mathrm{a}$ & $12,35 \mathrm{a}$ \\
\hline $\mathrm{Co} / \mathrm{S}$ & 12,2 & $140,4 \mathrm{c}$ & $12,53 \mathrm{c}$ & $12,9 \mathrm{c}$ & $107,2 \mathrm{~b}$ & $9,40 \mathrm{~b}$ \\
\hline $\mathrm{Tr} / \mathrm{S}$ & 14,5 & $201,6 \mathrm{ab}$ & $14,85 \mathrm{~b}$ & $14,3 \mathrm{a}$ & $157,1 \mathrm{a}$ & $11,94 \mathrm{a}$ \\
\hline$C V, \%$ & 1,4 & 4,2 & 11,3 & 1,1 & 4,7 & 12,7 \\
\hline Interacción Sec * NT & $P=0,001$ & ns & ns & ns & ns & ns \\
\hline
\end{tabular}

Av: avena; S: soja; Ce: cebada; Co: colza; Tr: trigo.

Dentro de cada columna, valores seguidos por la misma letra no difieren estadísticamente según la prueba de Tukey $(\mathrm{P}<0,05)$. CV: coeficiente de variabilidad.

Values in the same column followed by the same letter do not differ statistically, according to Tukey's test $(\mathrm{P}<0.05)$. $\mathrm{CV}$ : variability coefficient 

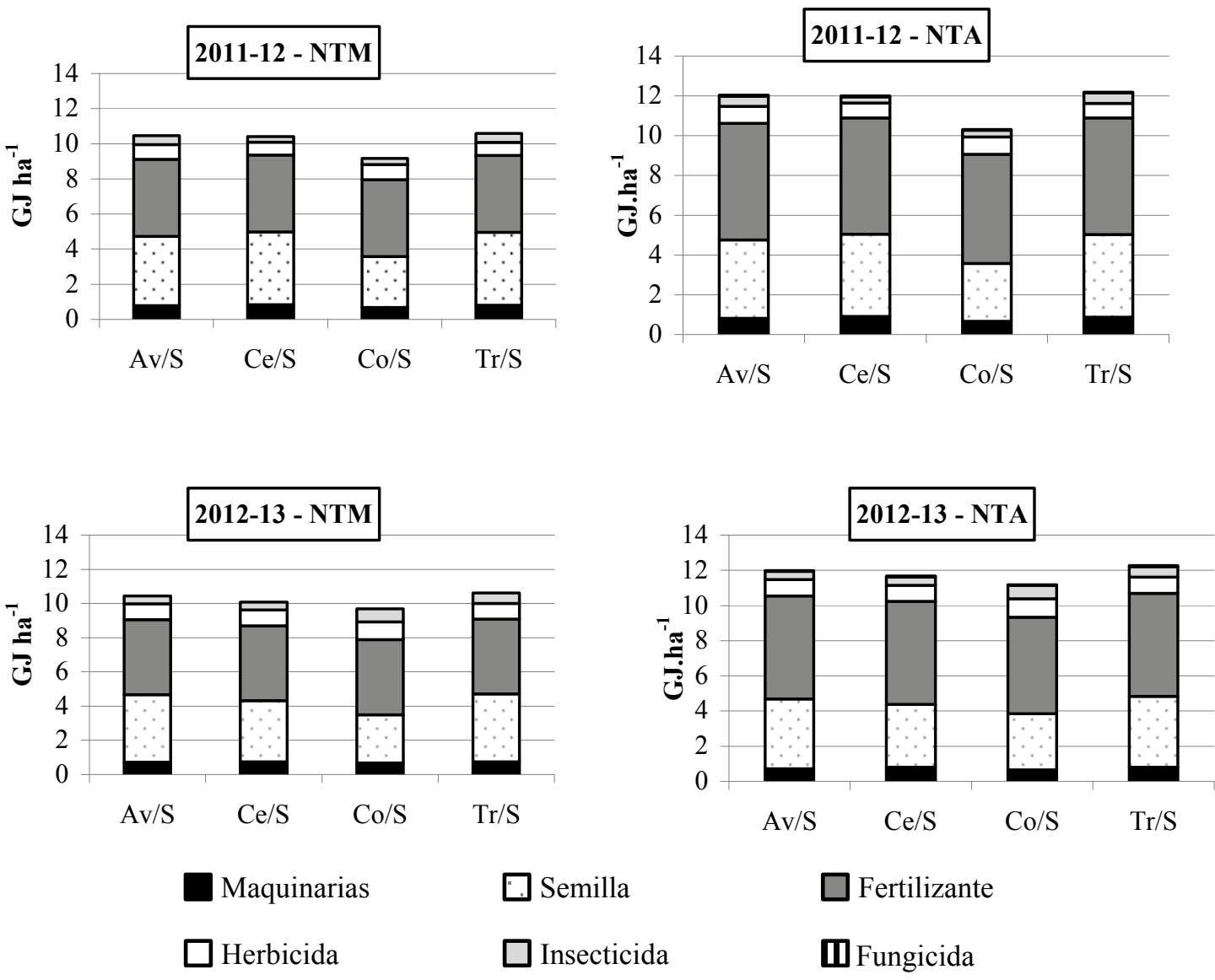

Fig. 1. Composición de la energía indirecta empleada para la producción de cuatro secuencias de cultivo en la provincia de Buenos Aires, Argentina, bajo dos modalidades de manejo en dos campañas.

NTM: nivel tecnológico medio; NTA: nivel tecnológico alto; A: avena; Ce: cebada; Co: colza; Tr: trigo; S: soja.

Fig. 1. Share of indirect energy used in the production of four crop sequences under two levels of technological management in two seasons, Buenos Aires province (Argentina).

NTM: medium technological level; NTA: high technological level. A: oat; Ce: barley; Co: rapeseed; Tr: wheat; S: soybean.

géticos de las semillas, y también al impacto de la energía relacionada con la fabricación de las maquinarias usadas para la cosecha y los diferentes rendimientos obtenidos.

La energía directa representó un 23\% del IE a los cultivos de invierno. La energía indirecta estuvo compuesta principalmente por los fertilizantes, que en el NTM y en los cereales promedió $75 \%$, ascendiendo al $80 \%$ en el NTA pero, en el caso de la colza, representaron mayores porcentajes, del 86 y el $89 \%$ en el NTM y NTA, respectivamente. En los cereales, el segundo insumo en costo energético fueron las semillas ( $13 \%$ de la energía indirecta), que en la colza fue sólo del $2 \%$ debido a su baja densidad de siembra. El tercer insumo en gasto energético fueron las maquinarias, con un valor promedio del $8 \%$. Las características de los años hicieron que no se requirieran aplicaciones de insecticidas, y los fungicidas, que sólo se usaron en el NTA no alcanzaron el 0,01\% del costo energético total. Sólo en la colza el gasto asociado a los herbicidas promedió el $2 \%$, mientras que en los cereales este valor fue despreciable.

Tanto los BE como la EE fueron notablemente menores en la campaña 2012-13 que en la anterior, correspondiendo los mayores valores a cebada y trigo (Tabla 3). 
Tabla 3. Ingreso de energía (IE), balance de energía (BE) y eficiencia energética (EE) para cuatro cultivos de invierno, promedio de dos manejos tecnológicos (NT), dos temporadas, en la provincia de Buenos Aires, Argentina.

Table 3. Energy input (IE), energy balance (BE) and energetic efficiency (EE) for four crops and average value for two levels of technological management (NT) in two seasons, Buenos Aires province, Argentina.

\begin{tabular}{|c|c|c|c|c|c|c|}
\hline \multirow[t]{2}{*}{ Cultivo } & \multicolumn{3}{|c|}{ 2011-12 } & \multicolumn{3}{|c|}{ 2012-13 } \\
\hline & IE & BE & $\mathrm{EE}$ & IE & BE & EE \\
\hline & \multicolumn{3}{|c|}{------- GJ ha'-1 ------ } & \multicolumn{2}{|c|}{--------GJ ha-1 ------- } & \\
\hline Avena & 8,4 & $118,8 \mathrm{~b}$ & $15,07 \mathrm{bc}$ & 8,0 & $53,1 \mathrm{c}$ & $7,68, \mathrm{c}$ \\
\hline Cebada & 9,1 & $157,9 \mathrm{a}$ & 18,36 a & 8,4 & $79,8 \mathrm{~b}$ & $10,61 \mathrm{~b}$ \\
\hline Colza & 7,1 & $86,3 \mathrm{c}$ & $13,20 \mathrm{c}$ & 7,1 & $53,7 \mathrm{c}$ & $8,65 \mathrm{bc}$ \\
\hline Trigo & 8,9 & $149,4 \mathrm{a}$ & $17,70 \mathrm{ab}$ & 8,6 & 105,1 a & $13,28 \mathrm{a}$ \\
\hline $\mathrm{CV} \%$ & 1,9 & 4,8 & 13,3 & 1,2 & 5,5 & 15,3 \\
\hline Interacción Cult * NT & $P=0,0000$ & ns & ns & $P=0,01$ & ns & ns \\
\hline
\end{tabular}

Dentro de cada columna, valores seguidos por la misma letra no difieren estadísticamente según la prueba de Tukey $(\mathrm{P}<0,05)$. CV: coeficiente de variabilidad.

Values in the same column followed by the same letter do not differ statistically, according to Tukey's test $(\mathrm{P}<0.05)$. $\mathrm{CV}$ : variability coefficient.

Tabla 4. Ingreso de energía para la producción de cuatro cultivos de invierno producidos bajo dos manejos tecnológicos, en dos temporadas, en la provincia de Buenos Aires, Argentina.

Table 4. Energy input in the production of four winter crops produced under two levels of technological management in two seasons, Buenos Aires province, Argentina.

\begin{tabular}{|c|c|c|c|c|c|c|}
\hline \multirow[t]{3}{*}{ Cultivo } & \multicolumn{3}{|c|}{ 2011-12 } & \multicolumn{3}{|c|}{ 2012-13 } \\
\hline & \multirow{2}{*}{$\begin{array}{l}\text { Energía } \\
\text { directa }\end{array}$} & \multicolumn{2}{|c|}{ Energía indirecta } & \multirow{2}{*}{$\begin{array}{c}\text { Energía } \\
\text { directa }\end{array}$} & \multicolumn{2}{|c|}{ Energía indirecta } \\
\hline & & NTM & NTA & & NTM & NTA \\
\hline & ------ & - & ------ & & & "--_-' \\
\hline Avena & $2,01 \mathrm{~b}$ & $5,68 \mathrm{f}$ & $7,12 \mathrm{c}$ & $1,67 \mathrm{c}$ & $5,66 \mathrm{f}$ & $7,06 \mathrm{c}$ \\
\hline Cebada & $2,33 \mathrm{a}$ & $6,09 \mathrm{~d}$ & $7,50 \mathrm{a}$ & $1,88 \mathrm{~b}$ & $5,79 \mathrm{e}$ & $7,19 \mathrm{~b}$ \\
\hline Colza & $1,57 \mathrm{c}$ & $5,03 \mathrm{~g}$ & $6,05 \mathrm{~d}$ & $1,53 \mathrm{~d}$ & $5,08 \mathrm{~g}$ & $6,10 \mathrm{~d}$ \\
\hline Trigo & $2,52 \mathrm{a}$ & $5,96 \mathrm{e}$ & $7,41 \mathrm{~b}$ & $2,05 \mathrm{a}$ & $5,81 \mathrm{e}$ & $7,24 \mathrm{a}$ \\
\hline $\mathrm{CV} \%$ & 6,3 & & & 4,1 & & \\
\hline $\begin{array}{l}\text { Interacción } \\
\text { Cult*NT }\end{array}$ & ns & & & ns & & \\
\hline
\end{tabular}

Para cada factor, valores seguidos por la misma letra no difieren estadísticamente según la prueba de Tukey $(\mathrm{P}<0,05)$. $\mathrm{CV}$ : coeficiente de variabilidad.

NTM: nivel tecnológico medio, NTA: nivel tecnológico alto.

For each factor, values followed by the same letter do not differ statistically, according to Tukey's test $(\mathrm{P}<0.05)$. CV: variability coefficient

NTM: medium technological level, NTA: high technological level.

Los flujos de energía en la soja

El IE para la producción de soja fue notablemente más reducido que el necesario para sus antecesores (Tabla 5). La energía directa necesaria fue menor tanto en términos absolutos, debido a que la soja se implantó bajo siembra directa, como por su participación en el IE (17\%). Respecto de la energía indirecta, la mayor contribución la hizo la semilla, con un $65 \%$ de la misma. El segundo insumo en importancia fueron los herbicidas (18\%), y luego los insecticidas (10\%).

En la campaña 2011-12 la soja registró diferentes IE según los distintos antecesores, pero con un rango bastante estrecho (Tabla 5). En 2012-13 esta variable registró interacción Antecesor x NT significativa, pero en ambos NT el menor IE lo regis- 
Tabla 5. Ingreso de energía (IE), balance de energía (BE) y eficiencia energética (EE) para soja sucediendo a cuatro cultivos de invierno, promedio de dos manejos tecnológicos (NT), en dos temporadas, en la provincia de Buenos Aires, Argentina.

Table 5. Energy input (IE), energy balance (BE) and energetic efficiency (EE) for soybean succeeding four winter crops, average value for two levels of technological management (NT), in two seasons, Buenos Aires province, Argentina.

\begin{tabular}{|c|c|c|c|c|c|c|}
\hline \multirow[t]{2}{*}{ Antecesor } & \multicolumn{3}{|c|}{ 2011-12 } & \multicolumn{3}{|c|}{$2012-13$} \\
\hline & IE & $\mathrm{BE}$ & $\mathrm{EE}$ & IE & $\mathrm{BE}$ & EE \\
\hline & ------ & 1 ------- & & ------ ( & ------- & \\
\hline Avena & $5,8 \mathrm{a}$ & $70,6 \mathrm{ab}$ & $13,10 a b$ & 5,8 & $70,6 \mathrm{ab}$ & $13,09 a b$ \\
\hline Cebada & $5,4 \mathrm{c}$ & $76,4 \mathrm{a}$ & 15,24 a & 5,4 & $76,4 \mathrm{a}$ & 15,09 a \\
\hline Colza & $5,1 \mathrm{~d}$ & $54,2 \mathrm{bc}$ & $11,58 \mathrm{~b}$ & 5,8 & $53,5 \mathrm{bc}$ & $10,34 \mathrm{bc}$ \\
\hline Trigo & $5,6 \mathrm{~b}$ & $52,2 \mathrm{c}$ & $10,33 \mathrm{~b}$ & 5,8 & $52,0 \mathrm{c}$ & $10,00 \mathrm{c}$ \\
\hline $\mathrm{CV} \%$ & 1,6 & 6,5 & 17,1 & 1,6 & 6,5 & 17,3 \\
\hline Interacción Ant * NT & ns & ns & ns & $P=0,01$ & ns & ns \\
\hline
\end{tabular}

Dentro de cada columna, valores seguidos por la misma letra no difieren estadísticamente según la prueba de Tukey $(\mathrm{P}<0,05)$. CV: coeficiente de variabilidad.

Values in the same column followed by the same letter do not differ statistically, according to Tukey's test $(\mathrm{P}<0,05)$. $\mathrm{CV}$ : variability coefficient

tró la soja que sucedió a cebada.

Los BE alcanzaron casi los mismos valores en las dos campañas. Su contribución al BE de la secuencia fue mayor en la campaña 2012-13 (45\%) que en 2011-12 (34\%). Tanto el mayor BE como la mayor EE los obtuvo la soja que sucedió a cebada y los menores con trigo como antecesor.

\section{Los flujos de energía según los manejos tecno- lógicos}

El IE a las secuencias de cultivos aumentó significativamente en el NTA respecto del NTM en la campaña 2012-13. En la campaña 2011-12 también aumentó, pero registró interacción NT $x$ Secuencia de cultivo significativa, asociada al consumo de energía indirecta y con poco significado biológico (Tabla 6). Se debe destacar que las diferencias entre los dos manejos son reducidas, del orden de 1,5 GJ ha'-1. El mayor IE se registró tanto en los cultivos de invierno como en la soja.

Los BE tampoco difirieron significativamente entre manejos, aun cuando en la campaña 201112 la producción de los cultivos de invierno respondió al NT. La EE no fue diferente entre manejos en la campaña 2011-12, pero sí en 2012-13 relacionándose inversamente con el IE (Tabla 6).

\section{DISCUSIÓN}

Los datos presentados muestran que en los sistemas de producción evaluados, los flujos de energía y la eficiencia con que es usada varían fundamentalmente entre cultivos y secuencias de cultivos, y que pueden ser afectados por la forma de producción y las condiciones de crecimiento.

La secuencia de cultivos que presentó el mejor comportamiento fue cebada/soja, ya que con un IE poco mayor a las restantes secuencias, obtuvo el mayor BE y la mayor EE en ambas campañas. La secuencia colza/soja, por otra parte, si bien fue la que requirió menos energía, alcanzó también muy bajos valores de BE y EE.

Estos resultados fueron consecuencia del comportamiento de los distintos cultivos. La cebada fue el cultivo más productivo y más eficiente $\mathrm{y}$, a la vez, por cosecharse temprano, brindó buenas condiciones de crecimiento a la soja que, en ambas campañas, rindió más como su sucesora y fue más eficiente en el uso de la energía.

Por otro lado, se observó una clara diferencia entre la colza y los cereales, caracterizándose estos últimos por valores notablemente mayores tanto en el IE como en el BE y la EE. Según Iermanó y Sarandón (2009) la mayor EE sería indicativa de su mejor adaptación agroecológica a la zona. El bioma original de la región pampeana, en la que se encuentra la provincia de Buenos Aires, es el pastizal, compuesto principalmente por gramíneas. Si bien el trigo, la cebada y la avena son originarios de otros lugares, su similitud morfológica y funcional con el pastizal les otorgaría una mejor adaptación que a la colza y, por lo tanto, un mejor comportamiento energético. Lo mismo puede decirse de la soja, que también registró menores valores de IE, BE y, en muchas situaciones, de EE. Todo esto justificaría que la secuencia col$\mathrm{za} /$ soja haya sido superada por las secuencias que incluyeron gramíneas en las distintas variables 
Tabla 6. Ingreso de energía (IE), balance de energía (BE) y eficiencia energética (EE) para cuatro secuencias cultivos bajo dos manejos tecnológicos: medio (NTM) y alto (NTA), en la provincia de Buenos Aires, Argentina.

Table 6. Energy input (IE), energy balance (BE) and energetic efficiency (EE) for four crop sequences produced under two levels of management: medium technological level (NTM) and high technological level (NTA), Buenos Aires province, Argentina.

\begin{tabular}{|c|c|c|c|c|c|c|}
\hline \multirow[t]{2}{*}{ Manejo } & \multicolumn{3}{|c|}{ 2011-12 } & \multicolumn{3}{|c|}{ 2012-13 } \\
\hline & IE & BE & EE & IE & BE & EE \\
\hline & \multicolumn{2}{|c|}{------- GJ ha-1 ------ } & & \multicolumn{2}{|c|}{------- GJ ha-1 ----- } & \\
\hline NTM & 13,1 & $184,8 \mathrm{a}$ & $15,1 \mathrm{a}$ & $12,9 \mathrm{~b}$ & $137,9 \mathrm{a}$ & $11,65 \mathrm{a}$ \\
\hline NTA & 14,7 & $198,1 \mathrm{a}$ & $14,4 \mathrm{a}$ & $14,5 \mathrm{a}$ & $134,2 \mathrm{a}$ & $10,18 \mathrm{~b}$ \\
\hline $\mathrm{CV} \%$ & 1,4 & 4,2 & 11,3 & 1,1 & 4,7 & 12,7 \\
\hline Interacción NT * Sec & $P=0,001$ & ns & ns & ns & ns & $\mathrm{ns}$ \\
\hline
\end{tabular}

Dentro de cada columna, valores seguidos por la misma letra no difieren estadísticamente según la prueba de Tukey (P $<0,05)$; CV: coeficiente de variabilidad; ns: no significativo.

Values in the same column followed by the same letter do not differ statistically, according to Tukey's test $(\mathrm{P}<0.05)$; CV: variability coefficient; ns: non-significant.

que hacen al uso de la energía.

La presunta ventaja de la colza de favorecer la producción de la soja de segunda por cosecharse más temprano que el trigo y la avena, por un lado, no se verificó en las dos campañas, y por otro lado, no alcanzó para otorgarle a la soja un mejor comportamiento energético y compensar la propia baja EE y BE de la colza, aún con bajos IE. En parte esto se relaciona con que el mayor aporte a la secuencia, tanto en el IE como en el BE, correspondió a los cultivos de invierno.

Por otro lado, debe señalarse la estabilidad de la soja en comparación con sus antecesores, al punto que, en la primera campaña los cultivos de invierno registraron mejores EE (13-18) pero en la segunda la redujeron a valores de 7-13, por debajo incluso de los registrados por la soja (10-15). Esto confirma la plasticidad de esta oleaginosa, que es una de las características que contribuyó a su difusión en la Argentina.

A pesar de lo anterior, y coincidiendo con Moreno et al. (2011), es necesario considerar la necesidad de incorporar cultivos dentro de los sistemas productivos que, a pesar de tener un comportamiento energético regular, aporten otros beneficios. En este caso particular, han sido resaltadas las ventajas de la inclusión de la colza por facilitar el manejo de plagas, malezas y enfermedades difíciles de controlar en planteos productivos basados en cereales de invierno (Iriarte, 2009).

Los cultivos se diferenciaron también en el uso que hicieron de los diferentes insumos. Mientras que en los cultivos de invierno fueron muy importantes los fertilizantes que, en coincidencia con los antecedentes (Tzilivakis et al., 2005; Moreno et al., 2011; Zentner et al., 2011) participa- ron con porcentajes mayores al 70\% de la energía indirecta, en la soja cobraron mayor importancia las semillas y los plaguicidas. Esto, además de su importancia desde el punto de vista de la energía, afecta otros aspectos de la sustentabilidad. La baja o nula fertilización en la soja es una práctica habitual en la producción agrícola de la zona, debido a que en la soja con las técnicas habituales de fertilización se resiente la fijación biológica de nitrógeno (Salvagiotti et al., 2008). Esta decisión reduce el costo energético del cultivo favoreciendo la incorporación de un recurso (nitrógeno) de manera casi gratuita al sistema, pero atenta contra la conservación del suelo como recurso, ya que los elevados requerimientos de nitrógeno de la soja determinan, normalmente, balances de nitrógeno muy negativos.

Al respecto, Iermanó y Sarandón (2009) mostraron cómo, en este cultivo, se redujo drásticamente la eficiencia energética cuando se cambió el planteo de fertilización usado por los productores por un planteo de reposición de los nutrientes principales (N, P, K, S y Ca). Paralelamente, aun cuando en los cultivos invernales una buena parte del costo energético se asigna a la aplicación de fertilizantes, esta resulta insuficiente, aún en el planteo de mayor tecnología, para lograr balances de nutrientes cercanos a 0 (Golik et al., 2014b). Es así como decisiones que se toman basándose en aspectos económico-financieros se relacionan con la aparición de problemas ecológicos y/o sociales (Flores y Sarandón, 2004).

Estos resultados apoyan lo señalado previamente acerca de que una alta eficiencia energética puede relacionarse con efectos negativos sobre otros componentes dentro o fuera de los agroecosistemas, tales como erosión del suelo y disminu- 
ción de la materia orgánica edáfica (Moreno et al., 2011) o pérdida de fertilidad química (Iermanó y Sarandón, 2009) sustentando la necesidad de que la eficiencia energética se considere como un indicador más dentro de una evaluación más compleja de los agroecosistemas que integre distintos aspectos de la sustentabilidad.

Por otro lado, también se observó que la soja usó menos energía directa (combustible) por su implantación en siembra directa. Al respecto, Deike et al. (2008) y Moreno et al. (2011) observaron que el ahorro de combustible por la disminución de las labranzas se contrarrestó con los mayores requerimientos de combustible asociado al manejo de las malezas. Si bien el glifosato (ya que las variedades sembradas en la Argentina son resistentes al mismo) no es un herbicida particularmente costoso energéticamente, su uso continuado se ha relacionado con la aparición de resistencia en distintas malezas (Antoniou et al., 2010) lo que plantea la necesidad de incentivar otras estrategias de manejo de las mismas.

Con respecto al uso de plaguicidas, insumo importante en la soja pero no en los cultivos de invierno, Deike et al. (2008) han señalado que si bien desde el punto de vista energético no resultan un insumo costoso, adquieren importancia al reducir las pérdidas de producción incrementando el balance de energía y, por consiguiente, la eficiencia energética. En este sentido, si bien es importante su uso a fin de mejorar el uso de la energía, es igualmente importante reducirlo al mínimo para minimizar otras consecuencias ecológicas derivadas de su aplicación. Por lo anterior, cobra relevancia el desarrollo y la difusión de prácticas relacionadas con el manejo integrado de las distintas adversidades basadas en el rediseño y manipulación de la biodiversidad, que permitan aprovechar los servicios ecológicos provistos por ésta (Gliessman et al., 2007).

Con respecto a la tecnología de producción es necesario resaltar que los manejos aplicados fueron similares a los implementados por los productores, lo cual no implica que el NTA sea la forma de producción adecuada o recomendada en relación a la sustentabilidad, sino que, en gran medida, son decisiones tomadas en base a la relación costo-beneficio de la práctica. Esto explica que las diferencias en el ingreso de energía entre manejos sean relativamente bajas para las secuencias y también que los balances no difieran en gran medida. A pesar de esto se observó la tendencia a disminuir la EE con la mayor aplicación de insumos. Considerando que uno de los insumos más costosos es el fertilizante nitrogenado (aproximadamente el $40 \%$ de la energía indirecta para la secuencia), toda práctica que mejore la fijación biológica por parte de la soja (a través de la simbiosis con los rizobios), o la posibilidad de aportarlo a través de la utilización de leguminosas como cultivos acompañantes en los cultivos de invierno, podrían ser de utilidad para reducir su costo energético y, a la vez, aportaría otras ventajas al sistema, al incrementar la biodiversidad y reforzar las funciones de autorregulación, como podría ser una menor utilización de plaguicidas.

\section{CONCLUSIONES}

Las secuencias (rotaciones) avena/soja, cebada/soja, colza/soja y trigo/soja producidas en la provincia de Buenos Aires (Argentina) muestran diferentes flujos energéticos. Los mayores ingresos de energía se registran en avena/soja y trigo/ soja y el menor en colza/soja. La energía directa (combustible) representa un $23 \%$ del ingreso total de energía, y dentro de la energía indirecta, los insumos más costosos son los fertilizantes y las semillas. Los balances energéticos y la eficiencia energética de las secuencias cebada/soja y trigo/ soja son mayores a los de avena/soja y colza/soja.

En el contexto de la experiencia, el mejor comportamiento lo registra la secuencia cebada/soja, que logra alta eficiencia energética y balance de energía con gastos energéticos sólo un poco más altos que las otras secuencias evaluadas.

El manejo tecnológico aplicado tiene menor influencia sobre las variables energéticas que los cultivos que componen la secuencia y las condiciones de crecimiento.

Se considera importante evaluar integralmente estas opciones productivas incorporando otros indicadores de sustentabilidad ecológica como el balance de nutrientes y de carbono del suelo, y el impacto por el uso de plaguicidas.

\section{LITERATURA CITADA}

Altieri, M., y C.I. Nicholls. 2000. Bases agroecológicas para una agricultura sustentable. p. 13-43. En M. Altieri y C.I. Nicholls (eds.) Agroecología. Teoría y práctica para una agricultura sustentable. Programa de las Naciones Unidas para el Medio Ambiente. Red de Formación Ambiental para América Latina y el Caribe. Serie Textos Básicos para la Formación Ambiental. México.

Antoniou, M., P. Brack, A. Carrasco, J. Fagan, M. Habib, P. Kageyama, C. Leifert, R. Onofre Nodari, y W. Pengue. 2010. Soja transgénica ¿Sostenible? ¿Responsable? Resumen de los principales resultados. GLS Gemeinschaftsbank eG and ARGE Gentechnik-frei. 12 p. Disponible en http://www.gmwatch.org/ files/GMsoy_Sust_Respons_SUMMARY_ SPA_v1.pdf (Consulta 4 junio 2015). 
Borin, M., C. Menini, and L. Sartori. 1997. Effects of tillage systems on energy and carbon balance in north-eastern Italy. Soil and Tillage Research 40:209-226.

Chamorro, A.M., R. Bezus, S.I. Golik, y A.E. Pellegrini. 2014a. Evaluación de distintos cultivos antecesores para la soja de segunda en el noreste de la Provincia de Buenos Aires. Revista Agronómica del Noroeste Argentino 34(2):132-135.

Chamorro, A.M., R. Bezus, S.I. Golik, y A. Pellegrini. 2014b. Eficiencia de uso del agua de lluvia para distintas secuencias de cultivos en La Plata, Buenos Aires. p. 25-26. En Reunión Binacional Uruguay-Argentina de Agrometeorología y XV Reunión Argentina de Agrometeorología. 1-3 de Octubre. Piriápolis, Uruguay. UniRío Editora, Río Cuarto, Córdoba, Argentina.

Cortesse, F. 2009. Información técnica de trigo y otros cultivos de invierno. Publicación Miscelánea № 113. INTA, Estación Experimental Agropecuaria Rafaela, Santa Fe, Argentina.

Dalgaard, T., N. Halberg, and J.R. Porter. 2001. A model for fossil energy use in Danish agriculture used to compare organic and conventional farming. Agriculture, Ecosystems and Environment 87:51-65.

Deike, S., B. Pallut, B. Melander, J. Strassemeyer, and O. Christen. 2008. Long-term productivity and environmental effects of arable farming as affected by crop rotation, soil tillage intensity and strategy of pesticide use: A case-study of two long-term field experiments in Germany and Denmark. European Journal of Agronomy 2:191-199.

Donato, L.B., I.R. Huerga, y J.A. Hilbert. 2008. Balance energético de la producción de biodiesel a partir de soja en la República Argentina. Documento № IIR-BC-INF-08-08 - INTA Bioenergía, Informes técnicos, año 2008, Boletín № 8. Disponible en http//www.inta.gov. ar/info/bioenergia/boletineslbc-inf-08-08.pdf (Consulta 3 julio 2010).

Donato, L.B., M.O. Tesouro, y A.A. Onorato. 2006. Costo Maq Versión 1.1. Software para la gestión integral de la maquinaria agrícola. INTA, Instituto de Ingeniería Rural - CIA, Castelar, Buenos Aires, Argentina.

FAO. 2011. Energy-smart food for people and climate. Issue paper. Food and Agriculture Organization of the United Nations (FAO). Available at http://www.fao.org/docrep/014/ i245e/i245e00.pdf. (Accessed September 2013).
Flores, C.C., and S.J. Sarandón. 2004. Limitations of neoclassical economics for evaluating sustainability of agricultural systems: comparing organic and conventional systems. Journal of Sustainable Agriculture 24(2):77-91.

Gliessman, S.R., F.J. Rosado-May, C. Guadarrama-Zugasti, J. Jedlicka, A. Cohn, V.E. Mendez, et al. 2007. Agroecología: promoviendo una transición hacia la sostenibilidad. Ecosistemas 16(1):13-23.

Golik, S.I., A.M. Chamorro, R. Bezus, y A. Pellegrini. 2014a. Cultivos de cebada y avena como alternativas del trigo: producción e incidencia de enfermedades foliares bajo dos niveles de fertilización. Chilean Journal of Agricultural \& Animal Science (Ex Agro-Ciencia) 30(1):45-56.

Golik, S.I., A.M. Chamorro, R. Bezus, y A.E. Pellegrini. 2014b. Extracción y balance de nutrientes para distintas secuencias agrícolas en el noreste de la Provincia de Buenos Aires. Revista Agronómica del Noroeste Argentino 34(2):147-150.

Guzmán, G.I., and A.M. Alonso. 2008. A comparison of energy use in conventional and organic olive oil production in Spain. Agricultural Systems 98:167-176.

Hüllsbergen, K.J., B. Feil, S. Biermann, G.W. Rathke, W.D. Kalk, and W. Diepenbrock. 2001. A method of energy balancing in crop production and its application in a long-term fertilizer trial. Agriculture, Ecosystems and Environment 86(3):303-321.

Iermanó, M.J., y S.J. Sarandón. 2009. ¿Es sustentable la producción de agrocombustibles a gran escala? El caso del biodiesel en Argentina. Revista Brasileira de Agroecologia 4(1):417.

Iriarte, L. 2009. Colza: un poco de historia, situación actual y perspectivas. AgroBarrow 43:1214. Disponible en http://www.inta.gov.ar/barrow/info/documentos/agrobarrow $43 / 05 \% 20$ colza\%20un \%20poco\%20de\%20historia $\% 20$ situacion $\% 20$ actual $\% 20 \mathrm{y} \% 20$ perspectivas. pdf (Consulta 5 abril 2011)

Khaledian, M.R., J.C. Mailhol, P. Ruelle, I. Mubarak, and S. Perret. 2010. The impacts of direct seeding into mulch on the energy balance of crop production system in the SE of France. Soil \& Tillage Research 106(2):218226.

Kuesters, J., and J. Lammel. 1999. Investigations of the energy efficiency of the production of winter wheat and sugar beet in Europe. European Journal of Agronomy 11(1):35-43. 
Moreno M.M., C. Lacasta, R. Meco, and C. Moreno. 2011. Rainfed crop energy balance of different farming systems and crop rotations in a semi-arid environment: Results of a longterm trial. Soil \& Tillage Research 114(1):1827.

Nagy, C.N. 1999. Energy coefficients for agriculture inputs in Western Canada. Canadian Agricultural Energy End-Use Data Analysis Centre (CAEEDAC). Available at http:// www.csale.usask.ca/PDFDocuments/energyCoefficientsAg.pdf. (Accessed May 2010).

Pellegrini, A.E., A.M. Chamorro, R. Bezus, S.I. Golik, y A. Frías Calvo. 2014. Efecto de rotaciones con soja de segunda en La Plata. $p$. 1-5. En Actas del XXIV Congreso Argentino de la Ciencia del Suelo, II Reunión Nacional "Materia Orgánica y Sustancias Húmicas". 5-9 de Mayo. Bahía Blanca, Buenos Aires, Argentina. Asociación Argentina de la Ciencia del Suelo, Buenos Aires, Argentina.

Pengue, W. 2004. Producción agroexportadora e (in)seguridad alimentaria: El caso de la soja en Argentina. Revista Iberoamericana de Economía Ecológica 1:46-55.

Salvagiotti, F., K.G. Cassman, J.E. Specht, D.T. Walters, A. Weiss, and A. Dobermann. 2008. Nitrogen uptake, fixation and response to fertilizer $\mathrm{N}$ in soybeans: A review. Field Crops Research 108(1):1-13.
Sarandón, S.J., M.S. Zuluaga, R. Cieza, C. Gómez, L. Janjetic, y E. Negrete. 2006. Evaluación de la sustentabilidad de sistemas agrícolas de fincas en Misiones, Argentina, mediante el uso de indicadores. Agroecología 1:19-28.

Tomaso, J.C. 2011. Cebada cervecera en la Argentina. IDIA 21:210-216.

Tzilivakis, J., D.J. Warner, M. May, L.A. Lewis, and K. Jaggard. 2005. An assessment of the energy inputs and greenhouse gas emissions in sugar beet (Beta vulgaris) production in the UK. Agricultural Systems 85(2):101-109.

Zentner, R.P., G.P. Lafond, D.A. Derksen, C.N. Nagy, D.D. Wall, and W.E. May. 2004. Effects of tillage method and crop rotation on non-renewable energy use efficiency for a thin Black Chernozem in the Canadian Prairies. Soil and Tillage Research 77(2):125-136.

Zentner, R.P., P. Basnyat, S.A. Brandt, A.G. Thomas, D. Ulrich, C.A. Campbell, et al. 2011. Effects of input management and crop diversity on non-renewable energy use efficiency of cropping systems in the Canadian Prairie. European Journal of Agronomy 34(2):113123. 